\title{
In Situ SERS Detection of Ferbam on Spinach Surfaces
}

\author{
Chunrong Wang ${ }^{\star 1, a}$, Jinhui Zhao ${ }^{2, b}$, Shan Wang ${ }^{1, c}$, Guoqiang $\mathrm{Wu}^{1, \mathrm{~d}}$, \\ Xianzai Yan ${ }^{1, d}$ and Yanping Hong ${ }^{1, e}$ \\ ${ }^{1}$ School of Food Science and Engineering, Jiangxi Agricultural University, Nanchang, Jiangxi, \\ 330045, China \\ ${ }^{2}$ School of Engineering, Jiangxi Agricultural University, Nanchang, Jiangxi, 330045, China \\ acrwang07@126.com, bjhxiaocao@sina.com, \\ cshanwang99@126.com, ${ }^{\mathrm{d}}$ wgoing-651@163.com, ${ }^{\mathrm{e}}$ yanxianzi@126.com, ${ }^{\prime} y$ phong72@yahoo.com.cn
}

Keywords: SERS, Ferbam, spinach, branched gold nanoparticles

Abstract. In this study an in situ surface enhanced Raman spectroscopic (SERS) method was developed to rapidly and sensitively detect Ferbam on spinach leave surfaces directly without extracting them out. Ferbam was used to contaminate the surfaces of spinach leaves. Then branched gold nanoparticles (GNPs) colloids were deposited on the leave surface contaminated with pesticides and then air-dried for Raman measurements. The results show that Ferbam can be detected and discriminated in situ using the developed SERS method. The limits of detection (LODs) of ferbam was $0.3 \mathrm{mg} \mathrm{kg}^{-1}$ on fresh spinach leaves. The developed SERS method provides a simple, rapid, and sensitive way to monitor pesticides on plant surfaces for safe productions of commercial vegetables.

\section{Introduction}

Ferbam (Fer) is an bactericide widely applied in vegetables and fruits ${ }^{[1]}$. Ferbam residue in food have traditionally been monitored by high-performance liquid chromatography (HPLC) based methods ${ }^{[2]}$. However, before chromatography analysis, time consuming sample extraction, clean-up and pre-concentration of samples are always required for this technique. Therefore, it is difficult to detect Fer with one simple extraction method and instrument. Surface-enhanced Raman spectroscopy (SERS) is a highly sensitive technique utilizing Raman spectroscopy and nanotechnology , which can greatly enhance the weak Raman molecular "fingerprint" through the placement of the analyte on noble metal nanoscale-roughened surfaces ${ }^{[3]}$. SERS has been widely explored to detect pesticides ${ }^{[4,5]}$. Many studies compared with these SERS methods demonstrated the faster and more convenient sample prep of SERS as compared to HPLC ${ }^{[6]}$.

Up to now, only a few studies were reported on the in situ detection of Organophosphates, pyrethroids and neonicotinoids residues on several plant surfaces using homemade SERS substrates ${ }^{[7}$, ${ }^{8]}$. Therefore, further studies are urgently needed to investigate the in situ SERS methods for more pesticides residues on various plant surfaces. Here, we aim to develop a simple, rapid, and sensitive in situ SERS method to detect bactericide (Fer) on spinach leave surfaces using GNPs with different morphology. To the best of our knowledge, this is the first investigation of detecting Ferbam with no pretreatment on fresh spinach leaves using a branched GNPs substrate for in situ SERS method.

\section{Experimental section}

Chemicals and reagents. Ferbam (Fer) was obtained from Crescent Chemical Co. (Hauppauge, NY, USA). Hydrochloroauric acid trihydrate $(\mathrm{HAuCl} 143 \mathrm{H} 2 \mathrm{O})$, SDS, sodium citrate, and polyvinylpyrrolidone (PVP) were purchased from China Medicine (Group) Shanghai Chemical Reagent Corporation. Peptide (NH2-Leu-Aib-Trp-Ome)was synthesized by conventional solution-phase method by using a racemization-free fragmentation/condensation strategy ${ }^{[9]}$.

Synthesis of Gold Nanoparticles and Characterization. Spherical gold nanoparitcles were prepared using Frens' method ${ }^{[10]}$. Ginger-like gold nanoparticles were fabricated as our previous 
method $^{[11]}$. Branched gold nanoparticles were obtained as method ${ }^{[12]}$. All the products prepared above were separated by centrifugation (TGL-16G, Anting Scientific Instrument Factory, China), and washed with deionized water several times. Optical spectra were recorded using a SPECORD200 spectrophotometer (Analytik Jena AG, Germany). TEM images were recorded on a JEM-2010 microscopy operated at $200 \mathrm{kV}$.

SERS Detection of Ferbam in water ${ }^{[8]}$. In a typical preparation, $2 \mu \mathrm{L}$ of working standard solution of Fer $10 \mu \mathrm{g} \mathrm{mL}^{-1}$ ) was dropped on a piece of parafilm $(1 \times 1 \mathrm{~cm})$. Pure solvent (no Fer) was used as a negative control. After that, $2 \mu \mathrm{L}$ of $250 \mu \mathrm{g} \mathrm{mL}^{-1} \mathrm{Au}$ NPs was added into it and mixed by pipetting for $20 \mathrm{~s}$, and then $2 \mu \mathrm{L}$ mixture was transferred onto the gold coated microscope slide, then allowed to dry under room temperature for Raman measurement.

SERS Detection of Fer on spinach leaf surface. Fresh spinach leaves (Purity Organic Fuji) were bought form a local supermarket in Nanchang, China. Fer-exposed samples were made suing a spiking method as follows: $20 \mu \mathrm{L}$ of each concentration $\left(0.1,0.5,1.0,5.0 \mu \mathrm{g} \mathrm{mL} \mathrm{L}^{-1}\right.$, in water) of different Fer samples was transferred on spinach leaves, and then allowed to dry at room conditions. The Fer-exposed plant surface was about $1 \mathrm{~cm}^{2} .4 \mu \mathrm{L}$ of Au NPs were dropped on the Fer-exposed plant surface, mixed briefly with Fer on it by gentle pipetting for $20 \mathrm{~s}$, and then allowed to dry under room temperature. Raman spectra were collected and analyzed under a DXR Raman microscope(Thermo Fisher Scientific, Madison, Wis., U.S.A.). Tests were done in triplicates. OMNIC ${ }^{\mathrm{TM}}$ software version 9.1 was used to control the Raman instrument.

\section{Result and discussion}

GNPs had the diameter of about $40 \mathrm{~nm}$ (Fig.1 a). Ginger-like GNPs shown in Fig.1b had several tips (3-5), which might act as "hot spots" for SERS detection. Branched GNPs (Fig.1c) seemed to form a fishnet structure. These GNPs sol indicated different UV-vis spectra (Fig.1d), respectively.

The SERS fingerprint patterns of Fer on Au NPs was shown in Fig. 2. Sphecial GNPs and ginger-like GNPs exhibited weak peaks due to their weak interactions between the groups and GNPs. Branched GNPs indicated distinct characteristic peaks due to its strong interactions between the chemical groups and GNPs. The highest peak of Fer was observed at $1380 \mathrm{~cm}^{-1}$ which associated with the deformation of $\mathrm{CH}_{3}$ and stretching of $\mathrm{CN}$. In addition, there were other relatively stronger peaks, such as $934 \mathrm{~cm}^{-1}$ associated with the stretching of $\mathrm{CH} 3 \mathrm{~N}$ and $\mathrm{C}=\mathrm{S}, 1146 \mathrm{~cm}^{-1}$ with the rocking of CH3 and stretching of CN. Obviously, branched GNPs substrate exhibited the most effective enhancement function and its main SERS peaks showed the strongest Raman intensity. The crystal powder Raman spectrum of Fer has characteristic peak at $1385 \mathrm{~cm}^{-1}$. Benjamin et al. found that Fer SERS spectra had characteristic peak at $1377 \mathrm{~cm}^{-1}$ arising from the $v(\mathrm{C}-\mathrm{N})$ stretching mode coupled to the symmetric $\delta\left(\mathrm{CH}_{3}\right)$ motion due to gold nanorods as surface enhanced Raman spectroscopy substrates ${ }^{[13,14]}$. So the peak of $1380 \mathrm{~cm}^{-1}$ can be used as finger peak for trace analysis of Fer investigation.

Five different concentrations of Fer in deionized water were analyzed to determine the limits of detcction (LOD) and linear ranges using the optimized SERS method. The result (Fig. 3a) indicated that the concentration of Fer was positively correlated to peaks intensity at $1380 \mathrm{~cm}^{-1}$ in the SERS spectra. PLS was used to evaluate the quantitative capacity of the method and PLS plots were showen in Fig.3b. Fer suggested satisfactory quantitative response with high correlation coefficients (0.9715) and low Root Mean Square Error of Calibration (RMSEC: 0.957). This result indicated that it could be effective to detect Fer on/in food matrix using the SERS method because Fer with stronger binding with brached GNPs could compete with other weaker interactions with branched GNPs.

Fresh spinach leaves surfaces were spiked with $0.3 \mathrm{mg} \mathrm{kg}^{-1}$ Fer. Without GNPs and Fer, there was no Fer signal detected. Without extracting Fer, we developed an in situ method to investigate Fer on the spinach leaves surfaces by transporting GNPs onto the leave surfaces. After dropping GNPs on the leave surface, there were "coffee rings" formed on leaves due to unevenly distributing of GNPs, which can be clearly observed under microscope (Fig.4b). We selected the border of the rings and found they have consistent good signal. There were no significant background intereference observed on spinach 
leaves. The characteristic peaks of Fer at around $1381 \mathrm{~cm}^{-1}$ and $1146 \mathrm{~cm}^{-1}$ were shown in Fig.4a. compared with other in situ SERS methods ${ }^{[1]}$, our method applied the branched GNPs which was more easily available and sensitive. The developed method have great potential for practical monitoring of pesticides on plant surface.

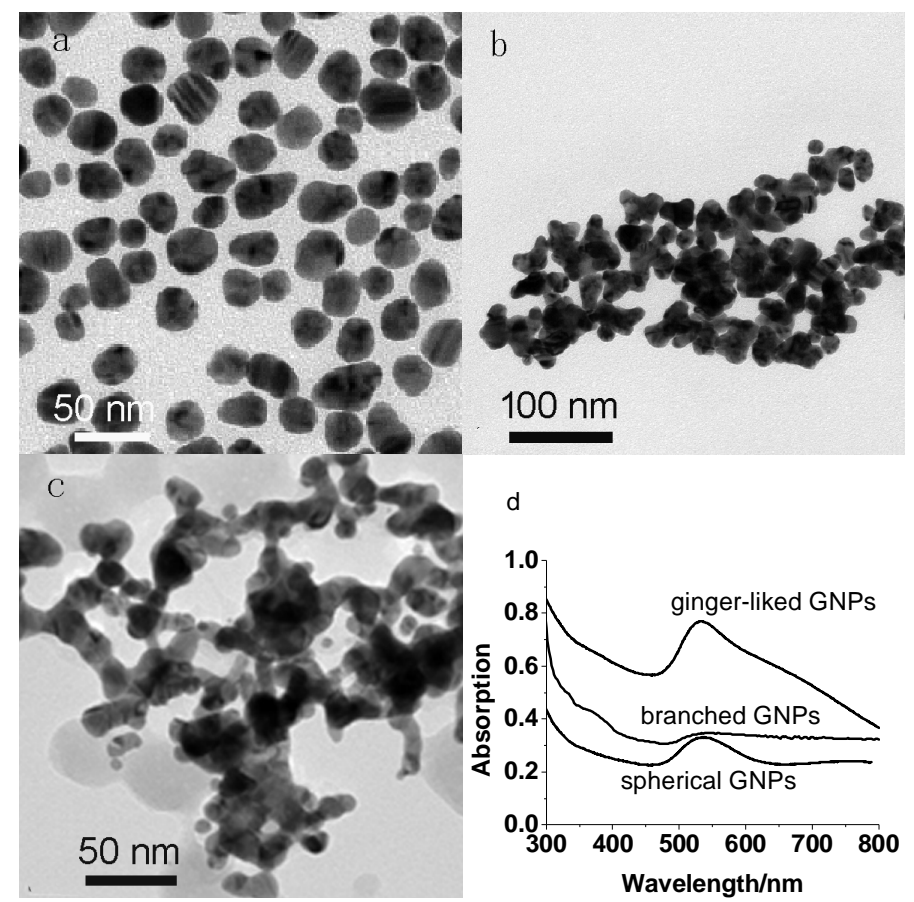

Fig.1 TEM images of (a)spherical GNPs; (b)ginger-like GNPs; (c) branched GNPs. (d) UV-vis spectra of different gold hydrosols.

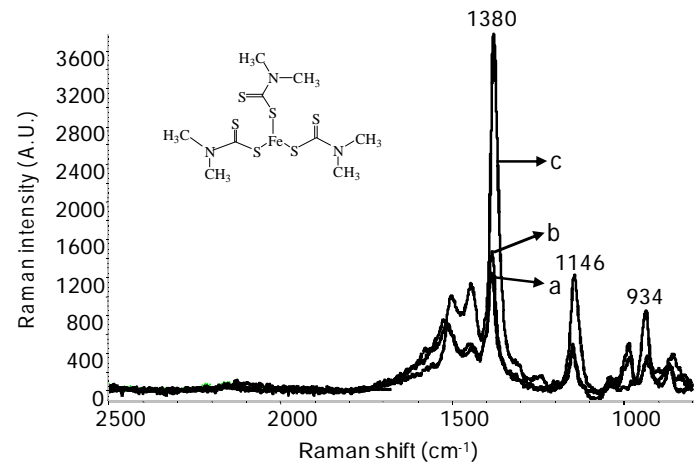

Fig. 2 SERS spectra of Fer from different gold nanoparticles substrates.
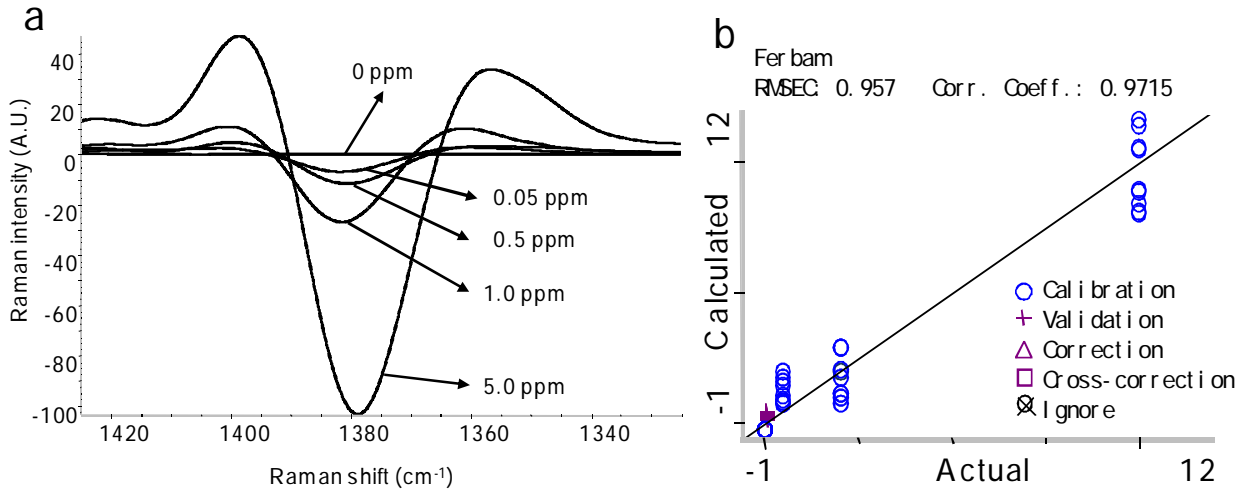

Fig.3 (a)second-derivative SERS spectra of characteristic peaks of different concentrations targets enhanced by branched GNPs. (b)PLS. 

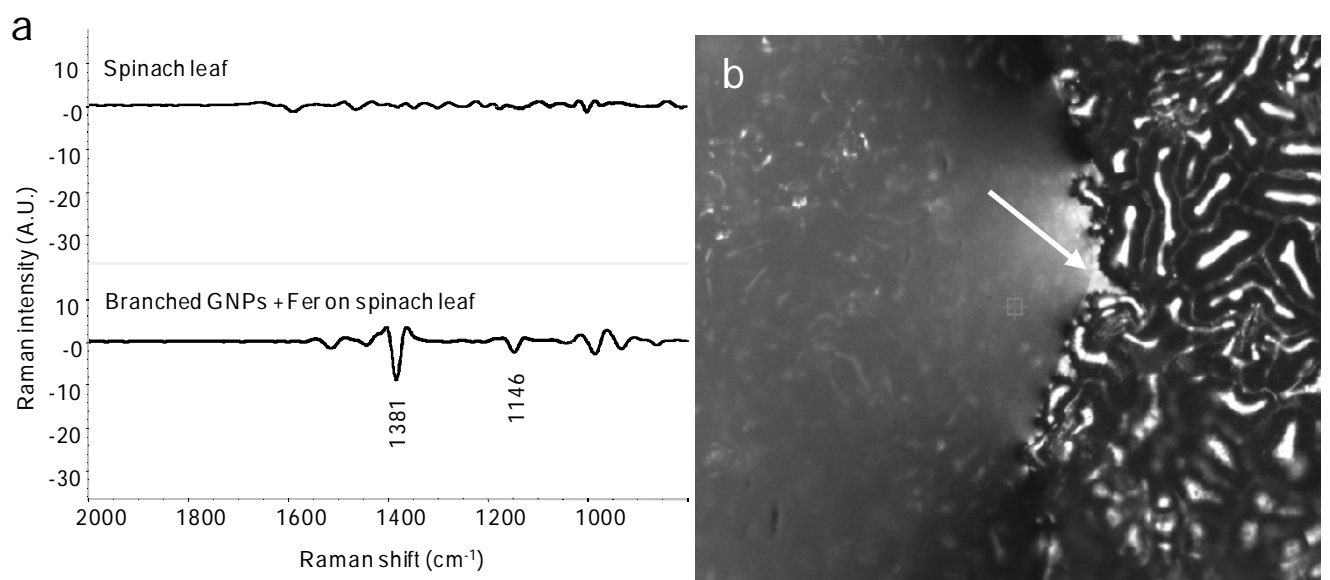

Fig. 4 (a)Raman spectra of Fer-exposed spinach leaf control, spiked level: $0.3 \mathrm{mg} \mathrm{kg}^{-1}(\approx 30 \mathrm{ng}$ $\mathrm{cm}^{-1}$ ); (b) Optical images of selected scan point. exposed-Fer spinach leaf was dropped with branched GNPs and mixed.

\section{Conclusions}

In summary, a simple and sensitive in situ SERS method was developed to detect ferbam on spinach leave surface. The proposed method can detect at least at $0.05 \mathrm{ppm}$ in water and $0.03 \mu \mathrm{g} \mathrm{cm}^{-1}$ on spinach leave surfaces. The established SERS method shows great capacity to analysis trace Fer residue on spinach surface. Further experiments are needed to explore the application of this method to other pesticides.

\section{Acknowledgements}

This work was financially supported by the Jiangxi provincial key research and development plan (20161BBG70074), the visiting scholarship grant from the China Scholarship Council (201308360013) and the National Science Foundation of China (grant no. 31000820).

\section{References}

[1] S. J. Rowan. CURR CANCER DRUG TAR,Vol.6(1979),p245

[2] A. L. García, E. B. González, A. Sanz-Medel. CHROMATOGRAPHIA,Vol.43(1996),p607

[3] R. Liu, S. Li, J. Liu. TrAC Trends in Analytical Chemistry,Vol.97(2017),p188

[4] S. Pang, T. Yang, L. He. Trac Trends in Analytical Chemistry,Vol.85(2016)

[5] S. Kumar, P. Goel, J. P. Singh. Sensors and Actuators B: Chemical,Vol.241(2017),p577

[6] M. B. Christiansen, E. Baadsgaard and J. Sanyova. Heritage Science,Vol.5(2017), p39

[7] T. Yang, Z. Zhang, B. Zhao, et al. ANAL CHEM,Vol.88(2016),p5243

[8] R. Hou, S. Pang, L. He. ANAL METHODS-UK,Vol.7(2015),p6325

[9] S. Si, T. K. Mandal. Chem. Eur. J.,Vol.13(2007),p3160

[10]G. Frens. NATURE,Vol.241(1973),p20

[11]C. R. Wang, Y. Fang, B. Li. ACTA PHYS-CHIM SIN,Vol.24(2008),p183

[12]C. R. Wang. Advanced Materials Research,Vol.638(2013),p2228

[13]H. Guo, Z. Zhang, B. Xing, et al. ENVIRON SCI TECHNOL,Vol.49(2015),p4317

[14]B. Saute, R. Narayanan. J RAMAN SPECTROSC,Vol.44(2013),p1518 